\title{
A Biomimic Reconstituted High Density Lipoprotein Nanosystem for Enhanced VEGF Gene Therapy of Myocardial Ischemia
}

\author{
Xiaotian Sun, ${ }^{1,2}$ Wenshuo Wang, ${ }^{1}$ Jiechun Huang, ${ }^{1,2}$ Hao Lai, ${ }^{1}$ \\ Changfa Guo, ${ }^{1}$ and Chunsheng Wang ${ }^{1}$ \\ ${ }^{1}$ Department of Cardiac Surgery, Zhongshan Hospital of Fudan University and Shanghai Institute of Cardiovascular Diseases, \\ 180 Fenglin Road, Shanghai 200032, China \\ ${ }^{2}$ Department of Cardiothoracic Surgery, Huashan Hospital of Fudan University, Shanghai 200040, China
}

Correspondence should be addressed to Changfa Guo; guo.changfa@zs-hospital.sh.cn and Chunsheng Wang; wang.chunsheng@zs-hospital.sh.cn

Received 7 December 2014; Accepted 18 March 2015

Academic Editor: Takuya Tsuzuki

Copyright (C) 2015 Xiaotian Sun et al. This is an open access article distributed under the Creative Commons Attribution License, which permits unrestricted use, distribution, and reproduction in any medium, provided the original work is properly cited.

\begin{abstract}
A biomimic reconstituted high density lipoprotein (rHDL) based system, rHDL/Stearic-PEI/VEGF complexes, was fabricated as an advanced nanovector for delivering VEGF plasmid. Here, Stearic-PEI was utilized to effectively condense VEGF plasmid and to incorporate the plasmid into $\mathrm{rHDL}$. The rHDL/Stearic-PEI/VEGF complexes with diameter under $100 \mathrm{~nm}$ and neutral surface charge demonstrated enhanced stability under the presence of bovine serum albumin. Moreover, in vitro cytotoxicity and transfection assays on H9C2 cells further revealed their superiority, as they displayed lower cytotoxicity with much higher transfection efficiency when compared to PEI 10K/VEGF and Lipos/Stearic-PEI/VEGF complexes. In addition, in vivo investigation on ischemia/reperfusion rat model implied that $\mathrm{rHDL} /$ Stearic-PEI/VEGF complexes possessed high transgene capacity and strong therapeutic activity. These findings indicated that rHDL/Stearic-PEI/VEGF complexes could be an ideal gene delivery system for enhanced VEGF gene therapy of myocardial ischemia, which might be a new promising strategy for effective myocardial ischemia treatment.
\end{abstract}

\section{Introduction}

In spite of increasing efforts to improve its management, cardiovascular disease (CVD) continues to be the leading cause of death worldwide, accounting for almost $30 \%$ of all deaths [1]. Within this group of disorders, myocardial ischemia remains to be one of the major causes, which occurs when blood supply to the myocardium is reduced by stenosis or occlusion of a coronary artery [2]. To avoid severe consequences of ischemia in the heart, the idea of growing new blood vessel for increasing blood supply and insuring better heart function has been proposed [3]. Evidences have proved that angiogenic factors could promote vessel growth and restore blood supply to the myocardium, thus preserving left ventricular (LV) function and preventing left ventricular remodeling $[4,5]$. Therapies using vascular endothelial growth factor (VEGF) have demonstrated beneficial effects of relieving myocardial ischemia symptoms [6, 7]. Compared with gene therapy, VEGF administered as a natural recombinant human protein in vivo exhibited some disappointing results $[8,9]$, which were attributed, at least partially, to the short lived effect and high instability of the protein when injected as a bolus [10]. The most common technique for myocardial ischemia gene therapy has been the utilization of viral vectors to deliver VEGF plasmid into cardiomyocytes [11]. While viral gene therapy offers high transfection efficiencies, its clinical utility is limited by many disadvantages, including host immune responses, oncogenic potential, limitations in viral loading, and difficulty in largescale manufacturing. Owing to these reasons, the development of safer, nonviral methods for gene delivery gains its popularity [12]. 
Among various nonviral vectors, polyethyleneimine (PEI) has been treated as the gold standard in cationic gene delivery polymers, for it shows high transfection efficiency. However, the inherent cytotoxicity and rapid clearance from the blood induced by its highly positive charges limit its further application in vivo [13]. As a result, the fabrication of a PEI derived vector with high transfection efficiency and low cytotoxicity appears to be a challenging issue in successful application of VEGF in myocardial ischemia gene therapy.

High density lipoprotein (HDL) is one of the essential components of the lipid transport system, which has been well established to play a protective role against the development of CVD [14]. The major protein component $(\sim 70 \%)$ in HDL is apolipoprotein A-I (apoA-I), a highly $\alpha$ helical polypeptide $(28 \mathrm{kDa})$, which has been proved to have high affinity to the ABCA1 protein, ABCG-I, and scavenger receptor-BI (SR-BI) on the vascular and myocardial cells $[15,16]$. Endogenous HDL particles are of complete biodegradation and nonimmunogenicity. Reconstituted HDL (rHDL) is the synthetic form of the endogenous human HDL, and it possesses similar physicochemical properties. In the past decades, rHDL has been successfully developed as a gene carrier $[17,18]$, which displayed promising application potential in vivo.

In this study, an rHDL-based system was developed for effective VEGF delivery and gene therapy in myocardial ischemia model. Stearic-PEI was first synthesized and then employed to construct a lipophilic core of rHDL (Lipos/Stearic-PEI). The cationic Stearic-PEI was served to condense the VEGF plasmid to formulate Lipos/StearicPEI/VEGF complexes. Finally, functional protein apoA-I was introduced to eventually assemble the delivery system (rHDL/Stearic-PEI/VEGF complexes). As a biomimic delivery system, the rHDL/Stearic-PEI/VEGF complexes were expected to remain stable and reduce the cytotoxicity of PEI in vitro. Moreover, they should be able to exert high transfection efficiency and strong therapeutic effect in vivo due to the combination effect of PEI and rHDL.

\section{Materials and Methods}

2.1. Materials. Polyethyleneimine (PEI, Mw: $10 \mathrm{KDa}), 1-$ ethyl-3-(3-dimethylaminopropyl) carbodiimide (EDC), Nhydroxysuccinimide (NHS), stearic acid, sodium cholate, bovine serum albumin (BSA), and 3-[4,5-dimethylthiazol-2yl]-2,5-diphenyltetrazolium bromide (MTT) were purchased from Sigma Aldrich (St. Louis, MO). Soybean phospholipids (PC, purity 90\%) were obtained from Evonik Degussa China Co., Ltd. (Shanghai, China). Cholesterol and cholesteryl esters (CE) were purchased from Pharmacia Biotech (NJ, USA). The apoA-I was isolated from the industrial waste during production of albumin in our laboratory. All other reagents were of analytical grade and used without further purification.

2.2. Plasmid Preparation. The VEGF plasmid used in this study was prepared as described previously [19]. Briefly, plasmid carrying the VEGF-165 coding region under the control of the cytomegalovirus (CMV) immediate early promoter enhancer region and the chicken beta globulin intron (pCMV-VEGF) was created. The therapeutic gene was inserted into pCMV-lei based on the MluI and BamHI restriction sites. Plasmid preparation was performed by double $\mathrm{CsCl}$ gradient purification and purity was confirmed by spectrophotometer (Hitachi Japan) at A260/A280.

2.3. Cell Culture. H9C2 cells were purchased from the Cell Bank of Shanghai Institute of Biochemistry and Cell Biology, Chinese Academy of Sciences (Shanghai, China) and cultured in DMEM medium (Gibco, USA) supplemented with $10 \%$ FBS (HyClone, USA), $100 \mathrm{U} / \mathrm{mL}$ penicillin, and $100 \mu \mathrm{g} / \mathrm{mL}$ streptomycin (Gibco, USA) in a humidified atmosphere of $95 \%$ air $/ 5 \% \mathrm{CO}_{2}$ incubator at $37^{\circ} \mathrm{C}$. All experiments were performed on cells in the logarithmic phase of growth.

2.4. Synthesis of Stearic-PEI. Stearic-PEI was prepared by coupling the carboxylic groups of stearic acid with the secondary amine groups of PEI through amidation reaction. Stearic acid (17.09 mg, $0.06 \mathrm{mmol}$ ), EDC (34.34 mg, $0.18 \mathrm{mmol})$, and NHS $(20.61 \mathrm{mg}, 0.18 \mathrm{mmol})$ were charged into a $5 \mathrm{~mL}$ tube, dissolved in $2 \mathrm{~mL}$ of dimethyl sulfoxide (DMSO), and kept at room temperature for $30 \mathrm{~min}$ to activate the carboxyl groups of stearic acid. PEI $10 \mathrm{KDa}$ (PEI 10K, $100 \mathrm{mg}, 0.01 \mathrm{mmol}$ ) was dissolved in $5 \mathrm{~mL}$ of DMSO in a $10 \mathrm{~mL}$ flask. The activated stearic acid solution was then dropwise added to the PEI solution with magnetic stirring. The reaction was allowed to continue with the protection of argon at room temperature for $24 \mathrm{~h}$. The mixture was purified by repeated precipitation in diethyl ether. The raw product was further purified by dialysis in deionized water (MWCO $3500 \mathrm{Da}, 2 \mathrm{~L} \times 3)$ to remove the unreacted stearic acid, EDC, and NHS. The resulting solution was lyophilized to obtain Stearic-PEI. The chemical structure of Stearic-PEI was characterized by $1 \mathrm{H}$ NMR (Avance 300, Bruker, Germany).

\subsection{Preparation of $r H D L / S t e a r i c-P E I / V E G F$ Complexes}

2.5.1. Preparation of Lipos/Stearic-PEI. Thin-film dispersion method was employed to construct Lipos/Stearic-PEI. Briefly, $120 \mathrm{mg}$ of PC, $12 \mathrm{mg}$ of cholesterol, and $24 \mathrm{mg}$ of CE were dissolved in $2 \mathrm{~mL}$ of organic solvent (chloroform : methanol = $1: 1, \mathrm{v} / \mathrm{v}$ ), and the solvent of lipid solutions was evaporated with a rotary evaporator at $30^{\circ} \mathrm{C}$ until a thin film was formed. The trace solvent residue was finally removed with a stream of nitrogen gas. $500 \mu \mathrm{L}$ of Stearic-PEI solution $(20 \mathrm{mg} / \mathrm{mL})$, $50 \mu \mathrm{L}$ of sodium cholate solution ( $30 \mathrm{mg} / \mathrm{mL}$ in PBS buffer), and Tris buffer $(0.1 \mathrm{M} \mathrm{KCl}, 10 \mathrm{mM}$ Tris, $1 \mathrm{mM}$ EDTA, $\mathrm{pH}$ 8.0) were added to dissolve the thin film. The mixture was vortexed thoroughly for $5 \mathrm{~min}$, followed by ultrasonication using an ultrahomogenizer (JY92II, Ningbo, China) until a clear suspension was obtained. The dispersion was then filtered through a $0.22 \mu \mathrm{m}$ filter and dialyzed to remove the free sodium cholate $(\mathrm{MWCO} 7500 \mathrm{Da}, 2 \mathrm{~L} \times 3)$. Finally, the prepared Lipos/Stearic-PEI complexes were collected and stored at $4^{\circ} \mathrm{C}$ until further use. 
2.5.2. Preparation of Lipos/Stearic-PEI/VEGF Complexes. VEGF plasmid was dissolved in PBS buffer to obtain a final concentration of $0.1 \mathrm{mg} / \mathrm{mL}$ and then dropwise added to the above prepared Lipos/Stearic-PEI complexes with vortex to formulate Lipos/Stearic-PEI/VEGF complexes.

\subsubsection{Preparation of $r H D L / S t e a r i c-P E I / V E G F$ Complexes.} Lipos/Stearic-PEI/VEGF complexes were incubated with $100 \mu \mathrm{L}$ of apoA-I solution ( $30 \mathrm{mg} / \mathrm{mL}$ in PBS buffer) to form rHDL/Stearic-PEI/VEGF complexes under $600 \mathrm{rpm}$ stirring at $25^{\circ} \mathrm{C}$ for $8 \mathrm{~h}$. The prepared $\mathrm{rHDL} / \mathrm{Stearic}-\mathrm{PEI} / \mathrm{VEGF}$ complexes were obtained and stored at $4^{\circ} \mathrm{C}$ until further use.

2.6. Particle Size and Zeta Potential Measurement. The particle size and zeta potential of Lipos/Stearic-PEI/VEGF and rHDL/Stearic-PEI/VEGF complexes were measured in triplicate by dynamic light scattering (DLS) using a Malvern Zetasizer (Nano ZS-90, Malvern instruments, UK) at $25^{\circ} \mathrm{C}$ with $90^{\circ}$ scattering angle.

2.7. BSA Challenging Assay. PEI 10K/VEGF, Lipos/Stearic$\mathrm{PEI} / \mathrm{VEGF}$, and $\mathrm{rHDL} /$ Stearic-PEI/VEGF complexes were prepared and then incubated with BSA solution at the final BSA concentrations of $0.2,0.4,0.6$, and $0.8 \mathrm{mg} / \mathrm{mL}$ for $1 \mathrm{~h}$ at $37^{\circ} \mathrm{C}$. The alteration in turbidity at $350 \mathrm{~nm}$ was monitored with spectrophotometer [13].

2.8. Cytotoxicity. The cytotoxicity of the complexes was measured by MTT assay. The H9C2 cells were seeded in 96well plates at a density of $1 \times 10^{4}$ cells/well and incubated for $70-80 \%$ cell confluence. PEI 10K/VEGF, Lipos/StearicPEI/VEGF, or rHDL/Stearic-PEI/VEGF complexes containing various concentrations of PEI were cocultured with cells for $24 \mathrm{~h}$. After that, MTT solution $(20 \mu \mathrm{L}, 5 \mathrm{mg} / \mathrm{mL})$ was added and cells were further incubated for $4 \mathrm{~h}$ at $37^{\circ} \mathrm{C}$. After the medium was removed, DMSO $(150 \mu \mathrm{L})$ was added to each well. The absorption was measured at $570 \mathrm{~nm}$ using a Universal Microplate Reader (EL800, BIO-TEK Instruments Inc., USA). The cell viability was determined as a percentage relative to untreated control cells.

\subsection{In Vitro Transfection of VEGF. H9C2 cells were seeded} on 24 -well plates at a density of $5 \times 10^{4}$ cells/well. After $24 \mathrm{~h}$ of incubation, the culture media were replaced with serum-free media containing PEI 10K/VEGF, Lipos/Stearic-PEI/VEGF, or rHDL/Stearic-PEI/VEGF complexes. After $4 \mathrm{~h}$, the cells were washed with PBS three times to thoroughly remove the uninternalized complexes and cultured with complete medium for $48 \mathrm{~h}$. The cell culture media were collected after transfection and the amount of VEGF production and secretion was quantified using a VEGF ELISA kit (R\&D Systems; Minneapolis, MN) according to the manufacturer's protocol.

2.10. Rat Ischemia/Reperfusion Model. The rat ischemia/reperfusion model was produced according to previous report [20]. All animal experiments were conducted in compliance with our institutional and NIH guidelines for care and use of research animals. Male Sprague Dawley rats purchased from Shanghai Laboratory Animal Center (SLAC, China) were firstly anesthetized in an induction chamber delivering $4 \%$ isoflurane. Then animals underwent tracheal intubation for ventilation that was maintained during the procedure on an operating table equipped with a warm water pad $(2 \%$ isoflurane at a tidal volume of $2.0 \mathrm{~mL}$ and a respiration rate of 70 breaths per minute). A small incision was made in the 5th intercostal space and the ribs were spread to expose the chest cavity. The left lung was gently collapsed and retracted to visualize the heart using a wet $2^{\prime \prime} \times 2^{\prime \prime}$ gauze pad. After widely incising the pericardium, the left anterior descending artery (LAD) coronary artery was exposed and then blocked $2-3 \mathrm{~mm}$ distal from its origin with an 7/0 polypropylene suture. The suture was threaded through a $2 \mathrm{~cm}$ length of PE-50 tubing (Becton Dickinson; Franklin Lakes, NJ) and then removed $30 \mathrm{~min}$ after the ligation. Blanching of the myocardium and visible dyskinesia of the anterior wall of the left ventricle was observed to confirm successful ligation of the LAD. The rats were randomly assigned to one of four experimental groups: (1) ligation only (control, $n=6$ ); (2) injection of PEI 10K/VEGF complexes $(n=6)$; (3) injection of Lipos/Stearic-PEI/VEGF complexes $(n=6)$; and (4) injection of $\mathrm{rHDL} /$ Stearic-PEI/VEGF complexes $(n=6)$. After assignment, the suture was removed from the myocardium and $100 \mu \mathrm{L}$ PBS or complexes solution containing $25 \mu \mathrm{g}$ of plasmid DNA was injected in 4 sites of the myocardium (3 sites around the ischemic border zone and 1 site in the central infarct zone). The chest was closed in layers and the animal was allowed to recover under a warming lamp and given injections of pain alleviating medication (Buprenorphine) and antibiotics (Cefazolin). Only those rats with an EF equal to or below $55 \%$ (as determined by echocardiography) 2 days after surgery were included in the following study.

2.11. Echocardiographical Evaluation. The echocardiographic analysis was performed using a Vevo 770 high-resolution ultrasound system (Visualsonics, Toronto, Canada). Ejection fraction $(\mathrm{EF})$ measurement was performed to evaluate the left ventricular (LV) systolic function of the heart. The calculation followed Simpson's rule and was based on a parasternal long axis view and four parasternal short axis views at different levels of the LV. At the long axis view, the left ventricle length was measured from the aortic annulus to the endocardial border at the apex level in both diastole and systole. At the parasternal short axis view, the endocardium was traced at four different levels in both systole and diastole, to derive the areas required to obtain Simpson's value. All measurements were performed offline using dedicated Vevo 770 quantification software (Vevo 770 version 3.0.0) [21].

2.12. Histological Studies. Three weeks after surgery, animals were sacrificed and hearts were harvested for subsequent histological analysis. Harvested hearts were fixed and sliced in three $4 \mathrm{~mm}$ thick segments from apex to base. The hearts were dehydrated and embedded in paraffin. Sections $(5 \mu \mathrm{m})$ were cut from each slice. 
In order to quantify the small caliber vessel density and area, anti-caveolin- $1 \alpha$ antibody (diluted 1:50) was used as marker, and 2 peri-infarct and 2 intrainfarct images per section were analyzed. Secondary antibody was Alexa Fluor 488 goat conjugated anti-mouse IgG (diluted 1:100). Images were captured using the Axio Cam MR3 video camera at 20x connected to the Zeiss Axio Imager M1 microscope equipped with epifluorescence optics. Digital images were analyzed by MATLAB software platform (Mathworks Inc., Natick, MA, USA) [22].

The determination of apoptosis was performed by a commercially available ApopTag Fluorescein Apoptosis Detection Kit (Millipore; Billerica, MA) according to the manufacturer's instructions. Apoptosis in the border zone was imaged at 40x magnification using confocal laser scanning microscope (CLSM, Leica TCS SP5, Germany) and quantified by counting positively stained cells from five random high power fields for each animal.

\section{Results and Discussion}

3.1. Characterization of Stearic-PEI. The conjugation of stearic acid with PEI was conducted via amidation reaction. The cationic amido groups of PEI $10 \mathrm{~K}$ were employed to condense the VEGF plasmid; on the other hand, the highly hydrophobic stearic groups were introduced to incorporate the PEI/DNA complexes with the hydrophobic component of rHDL through hydrophobic interaction. Here, the StearicPEI served not only in packaging the VEGF plasmid, but also as a linker to integrate the DNA with rHDL. The chemical structure of Stearic-PEI was confirmed by ${ }^{1} \mathrm{H}$ NMR in $\mathrm{D}_{2} \mathrm{O}$. As shown in Figure 1, compared with the spectrum of PEI, the proton peaks of $-\mathrm{NHCH}_{2} \mathrm{CH}_{2}$ - from Stearic-PEI appeared at 2.4-3.4 ppm, whereas PEI only appeared at about $2.7 \mathrm{ppm}$. Moreover, the products had alkyl peaks of stearic acid at 0.82 , 1.18 , and $1.38 \mathrm{ppm}$. These results provided decisive evidences that stearic acid was successfully grafted to the PEI chain.

3.2. Particle Size, Zeta Potential Measurement, and BSA Challenging Assay. An ideal gene delivery system requires meticulous design of its particle size and zeta potential, as multiple researches have demonstrated that the cellular uptake of particles is in great relation to their size and surface charge $[23,24]$. Smaller size usually leads to preferable cellular uptake and superior therapeutic effect of particles, for they can be readily recognized and transported by corresponding receptor or channel [25]. Herein, the particle size and zeta potential of Lipos/Stearic-PEI/VEGF and rHDL/StearicPEI/VEGF complexes were analyzed. As shown in Table 1, both Lipos/Stearic-PEI/VEGF and rHDL/Stearic-PEI/VEGF complexes showed nanoscale size under $100 \mathrm{~nm}$. Comparing the particle size of these two complexes, a minor increase was observed in $\mathrm{rHDL} /$ Stearic-PEI/VEGF group, which indicated the successful coating of apoA-I protein. This conclusion was further confirmed by the significant change of zeta potential between them. It is well established that the positively charged particles tend to interact with negatively charged proteins in the blood and extracellular matrix, which

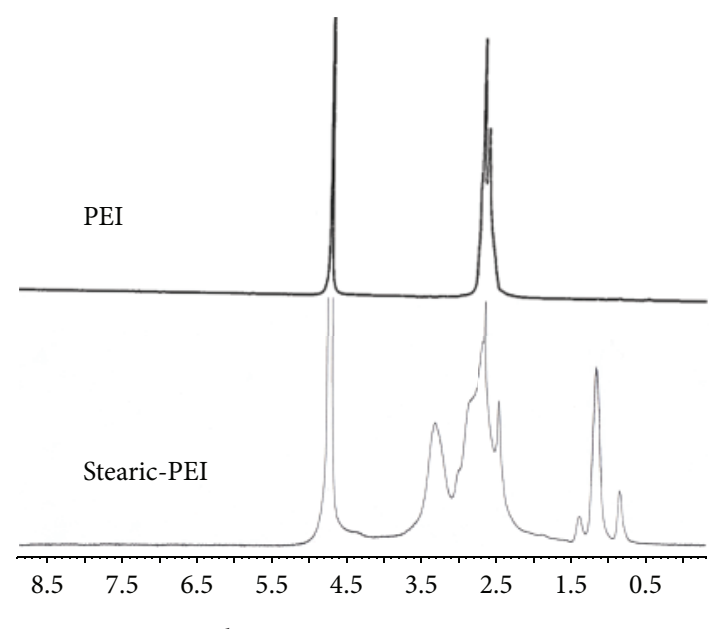

Figure 1: ${ }^{1} \mathrm{H}$ NMR of PEI and Stearic-PEI.

TABLe 1: Particle size and zeta potential of complexes.

\begin{tabular}{lcc}
\hline & $\begin{array}{c}\text { Particle size } \\
(\mathrm{nm})\end{array}$ & $\begin{array}{c}\text { Zeta potential } \\
(\mathrm{mV})\end{array}$ \\
\hline Lipos/Stearic-PEI/VEGF & $83.6 \pm 3.2$ & $+32 \pm 5.4$ \\
rHDL/Stearic-PEI/VEGF & $95.4 \pm 2.8$ & $-5.2 \pm 0.9$ \\
\hline
\end{tabular}

could be an obstacle for the effective transfection of VEGF plasmid; however, negatively charged particles are less likely to be taken up by the cells, for the cell membrane is also negatively charged and, also, it is a common rule that negative charged carrier has less stable complex with DNA [26]. To dissolve this dilemma, we employed layer-by-layer method to construct rHDL/Stearic-PEI/VEGF. The positively charged Lipos/Stearic-PEI was firstly introduced to condense VEGF plasmid; then the Lipos/Stearic-PEI/VEGF complexes were coated with apoA-I protein to shield the surface charge. In order to verify the superiority of the apoA-I coating in inhibiting serum protein interaction, the protein adsorption behavior of Lipos/Stearic-PEI/VEGF and rHDL/StearicPEI/VEGF complexes was explored with the presence of negatively charged BSA. As revealed in Figure 2, positively charged PEI 10K/VEGF and Lipos/Stearic-PEI/VEGF complexes exhibited serious protein adsorption profile with the increase of BSA concentration as predicted. On the contrary, rHDL/Stearic-PEI/VEGF complexes with neutral surface charge showed negligible change in turbidity, suggesting their safe application and high transfection potential in vivo.

3.3. Cytotoxicity. The safe issue should always be the primary concern of an ideal delivery system. As a result, the cytotoxicity of PEI 10K/VEGF, Lipos/Stearic-PEI/VEGF, and rHDL/Stearic-PEI/VEGF complexes was evaluated against H9C2 cells by MTT assay. Cells were treated with complexes containing various PEI concentrations ranging from 2 to $100 \mu \mathrm{g} / \mathrm{mL}$. As presented in Figure 3, significant inhibitory effects of PEI 10K/VEGF and Lipos/Stearic-PEI complexes were observed. PEI 10K/VEGF and Lipos/Stearic-PEI/VEGF complexes displayed $70.42 \%$ and $67.28 \%$ mortality on H9C2 cells at $100 \mu \mathrm{g} / \mathrm{mL}$, respectively, which might be related 


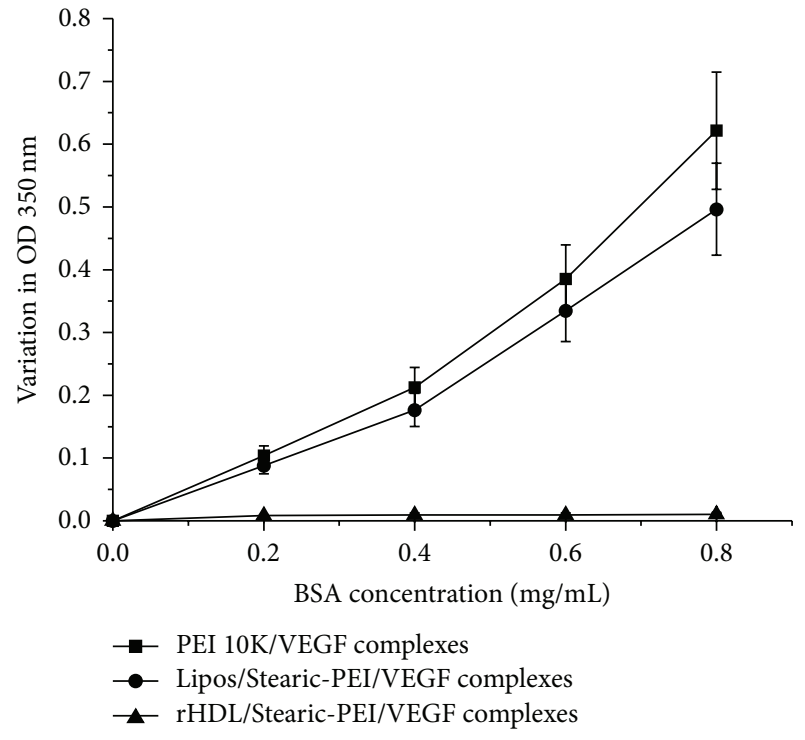

FIGURE 2: Stability of PEI 10K/VEGF, Lipos/Stearic-PEI/VEGF, and $\mathrm{rHDL} /$ Stearic-PEI/VEGF complexes in BSA. The variation in turbidity was measured at $350 \mathrm{~nm}$ after incubation with BSA for $1 \mathrm{~h}$. Data were expressed as mean \pm S.D. $(n=5)$.

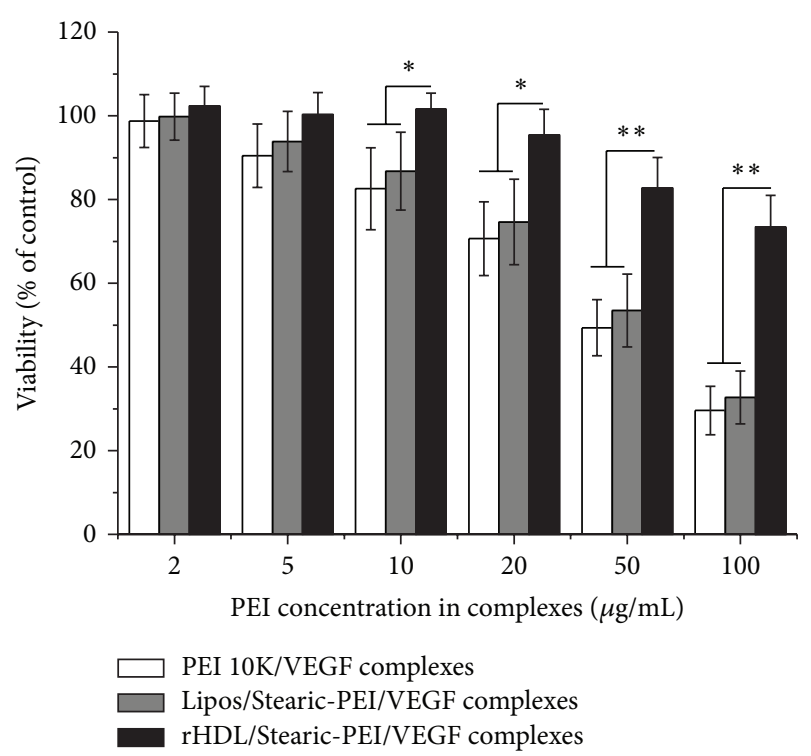

FIgUrE 3: Cell viability (\% of control) of PEI 10K/VEGF, Lipos/Stearic-PEI/VEGF, and rHDL/Stearic-PEI/VEGF complexes against H9C2 cells. Data were expressed as mean \pm S.D. $(n=5)$. ${ }^{*} P<0.05$ and ${ }^{* *} P<0.01$.

to their considerable positive charges. In contrast, compared with PEI 10K/VEGF and Lipos/Stearic-PEI/VEGF complexes in all groups, the cell viability of $\mathrm{rHDL} /$ StearicPEI/VEGF complexes was higher. At the PEI concentration of $100 \mu \mathrm{g} / \mathrm{mL}$, especially, the serious cytotoxicity of Lipos/Stearic-PEI/VEGF complexes was dramatically decreased after apoA-I protein shielding, indicating that rHDL as a biomimic delivery vector could indeed lower the

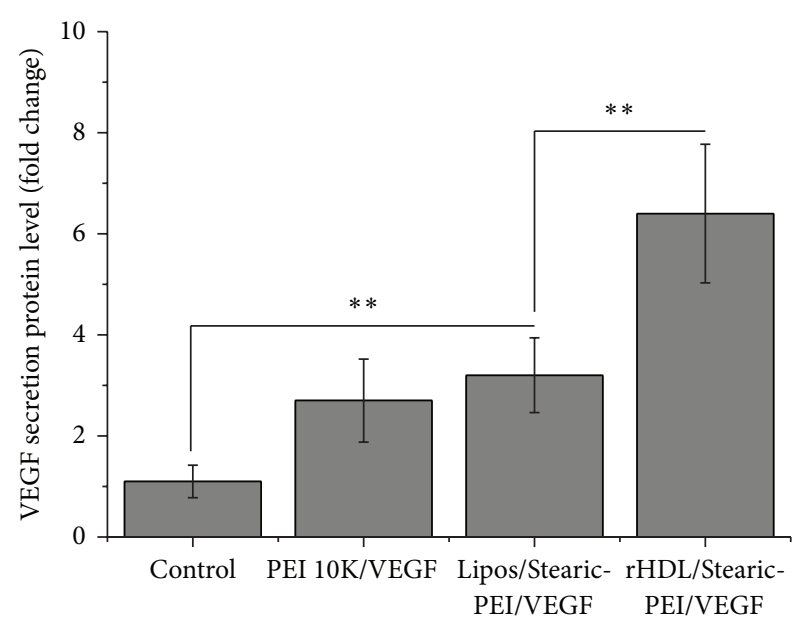

FIGURE 4: VEGF transfection of PEI 10K/VEGF, Lipos/StearicPEI/VEGF, and rHDL/Stearic-PEI/VEGF complexes in H9C2 cells. Data were expressed as mean \pm S.D. $(n=5) .{ }^{*} P<0.05$ and ${ }^{* *} P<0.01$.

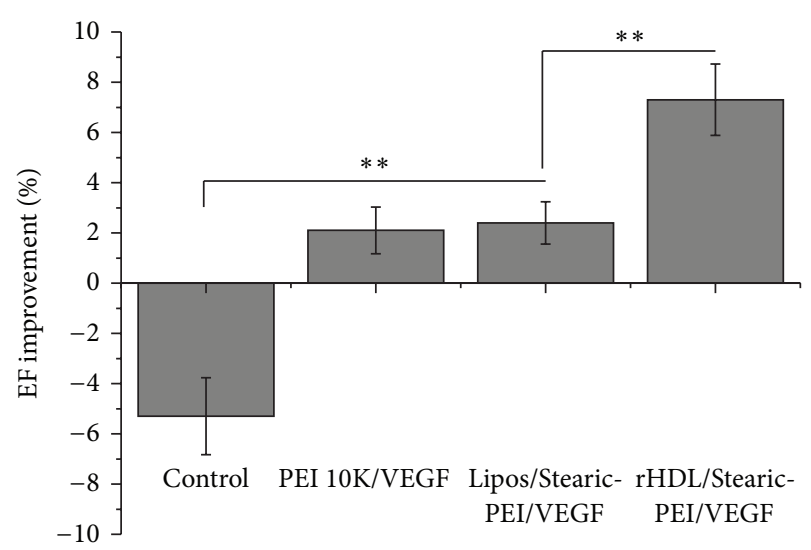

FIGURE 5: Ejection fraction (cardiac function) observed in different complexes. EF was assessed by echocardiography before and 3 weeks after treatment and mean improvement \pm S.D. $(n=6)$ is represented. ${ }^{*} P<0.05$ and ${ }^{* *} P<0.01$.

cytotoxicity of PEI, which was in line with our speculation in Section 3.2.

3.4. In Vitro Transfection of VEGF. HDL is one of the essential components of the lipid transport system with the ability to specifically bind with the SR-BI. As rHDL has been proved to possess similar properties to HDL, which can directly deliver their payload from rHDL to cytoplasm through nonaqueous "channel" of SR-BI [27], we anticipated that the VEGF plasmid in $\mathrm{rHDL} /$ Stearic-PEI/VEGF complexes can be internalized more effectively than that in PEI 10K/VEGF and Lipos/Stearic-PEI/VEGF complexes via the receptor mediated transportation. In order to verify our speculation, the transfection efficiency of the VEGF plasmid in H9C2 cells using PEI 10K/VEGF, Lipos/Stearic$\mathrm{PEI} / \mathrm{VEGF}$, and $\mathrm{rHDL} /$ Stearic-PEI/VEGF complexes was assessed by ELISA. The untreated cells were employed as a 


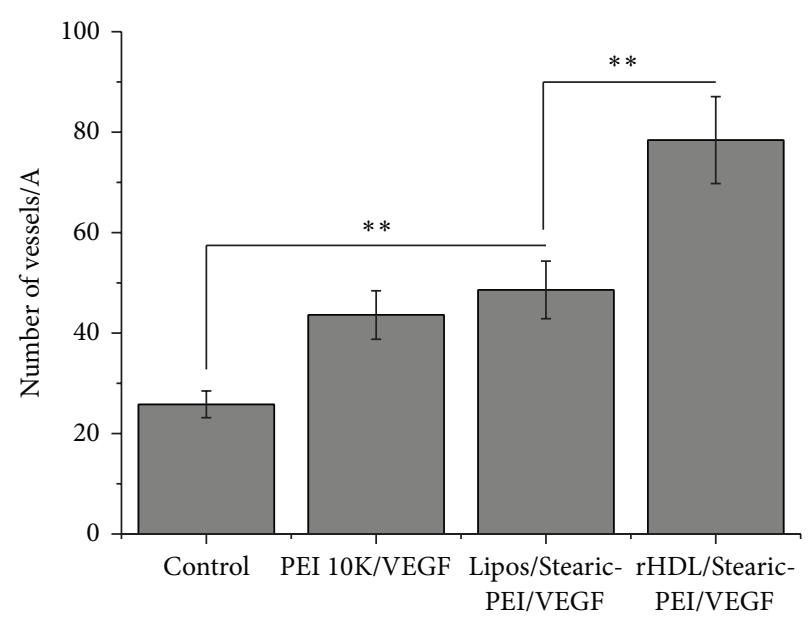

(a)

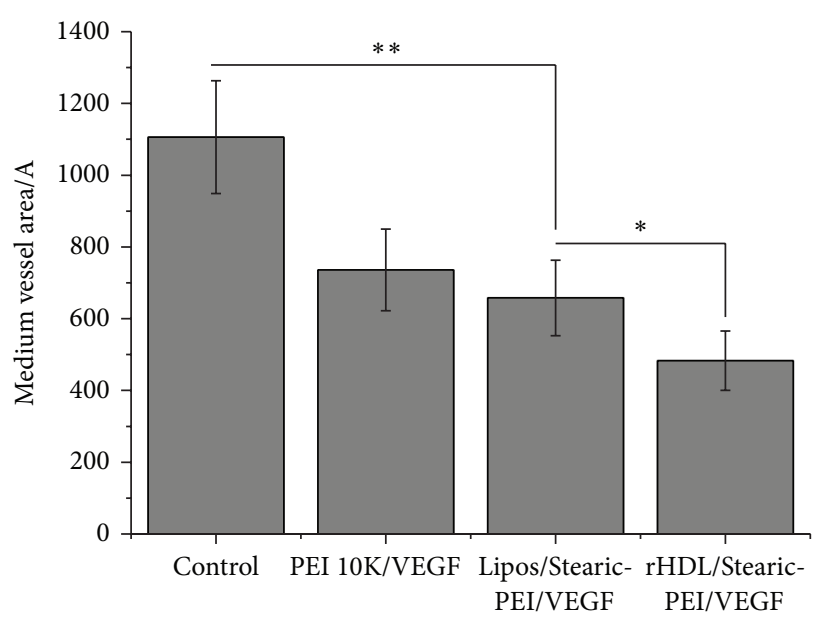

(b)

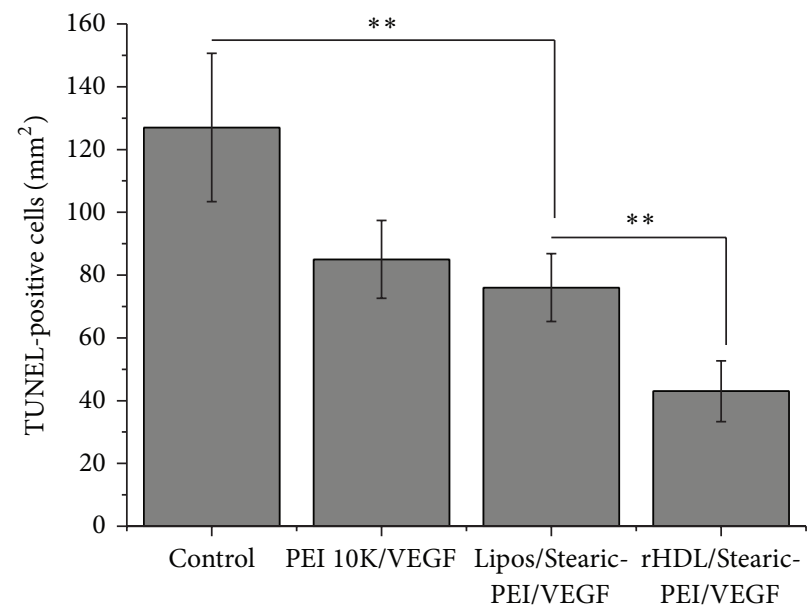

(c)

FIGURE 6: Histological analyses of PEI 10K/VEGF, Lipos/Stearic-PEI/VEGF, and rHDL/Stearic-PEI/VEGF complexes. (a) Vessel density, (b) average vessel diameter, and (c) the number of TUNEL-positive cells. Data were expressed as mean \pm S.D. $(n=6)$. ${ }^{*} P<0.05$ and ${ }^{* *} P<0.01$.

blank control with secretion of VEGF as 1-fold. As shown in Figure 4, the relative secretion of VEGF in PEI 10K/VEGF and Lipos/Stearic-PEI/VEGF complexes was 2.7- and 3.2-fold higher than that in control group, respectively, indicating that both PEI 10K/VEGF and Lipos/Stearic-PEI/VEGF complexes hold certain transfection efficiency. However, it was worth mentioning that rHDL/Stearic-PEI/VEGF complexes with apoA-I protein shielding displayed the most effective capacity among three complexes with a relative secretion level of 6.4fold, which was 2.4- and 2.0-fold higher than PEI 10K/VEGF and Lipos/Stearic-PEI/VEGF complexes, respectively. This finding provided decisive evidence that rHDL could indeed improve the transfection efficiency of PEI and the combination of them was proved to be more powerful than applying PEI alone. Based on the above results, effective transfection of rHDL/Stearic-PEI/VEGF complexes in vivo can be expected.

3.5. In Vivo Therapeutic Effect. The in vivo therapeutic effect of different formulations was evaluated by measuring the EF rates and performing histological assays on ischemia/ reperfusion rat model. As depicted in Figure 5, all formulations improved the heart function to some extent, among which rHDL/Stearic-PEI/VEGF complexes significantly restored the heart function as indicated by most increased EF rates when compared to other groups. In detail, rHDL/Stearic-PEI/VEGF complexes improved 7.32\% of the EF rates, which was 3.47- and 3.04-fold higher than that of PEI 10K/VEGF and Lipos/Stearic-PEI/VEGF complexes, respectively. The same conclusion could also be reached by the histological assays. Animals receiving $\mathrm{rHDL} /$ StearicPEI/VEGF complexes showed a statistically highly significant increase in the number of capillaries in the infarct and periinfarct area (Figure 6(a)). In addition, average vessel diameter analysis (Figure 6(b)) implied that these vessels were mostly neonatal ones, whose diameter was merely half of that in control group. Similar conclusion could also be drawn from the TUNEL assay. TUNEL-positive cells indicated the presence of apoptotic cardiomyocytes in the border zone of the infarct area (Figure 6(c)). The average number of TUNELpositive cardiomyocytes in five random regions was $\sim 127$ in the control group, $\sim 110$ in the PEI 10K/VEGF group, $\sim 76$ 
in the Lipos/Stearic-PEI/VEGF complexes treatment group, and $\sim 43$ in the rHDL/Stearic-PEI/VEGF complexes treatment group. The rHDL/Stearic-PEI/VEGF complexes treated hearts demonstrated the most remarkable reduction in apoptotic cardiomyocytes compared to both PEI 10K/VEGF and Lipos/Stearic-PEI/VEGF groups. All the above data indicated that the rHDL/Stearic-PEI/VEGF complexes with the combination of PEI and rHDL were the most potent formulation and the myocardial ischemia was greatly improved with the treatment of rHDL/Stearic-PEI/VEGF complexes.

\section{Conclusion}

In this work, a biomimic rHLD based gene delivery system, rHDL/Stearic-PEI/VEGF, was successfully developed with the aim of enhancing the efficacy of VEGF gene therapy of myocardial ischemia. The rHDL/Stearic-PEI/VEGF complexes containing PEI 10K were able to condense VEGF plasmid into nanosized particles with a diameter under $100 \mathrm{~nm}$. On the other hand, the biomimic structure of rHDL with similar properties to natural HDL demonstrated biocompatible and safe profiles in vitro. Transfection in H9C2 cells and in vivo therapeutic assays on ischemia/reperfusion rat model provided convincing evidences to the high performance of rHDL/Stearic-PEI/VEGF complexes. Taking all these data into account, we can conclude that $\mathrm{rHDL} /$ Stearic-PEI/VEGF complexes with biocompatible and potent transgene properties could be selected as a potential nonviral VEGF delivery system and new promising strategy for effective myocardial ischemia treatment.

\section{Conflict of Interests}

The authors report that they have no conflict of interests.

\section{Authors' Contribution}

Xiaotian Sun and Wenshuo Wang contributed equally to this work.

\section{References}

[1] A. Chockalingam, J. Chalmers, L. Lisheng et al., "Prevention of cardiovascular diseases in developing countries: agenda for action (statement from a WHO-ISH meeting in Beijing, October 1999)," Journal of Hypertension, vol. 18, no. 12, pp. 17051708, 2000.

[2] A. Kawamoto, H.-C. Gwon, H. Iwaguro et al., "Therapeutic potential of ex vivo expanded endothelial progenitor cells for myocardial ischemia," Circulation, vol. 103, no. 5, pp. 634-637, 2001.

[3] S. Mitsos, K. Katsanos, E. Koletsis et al., "Therapeutic angiogenesis for myocardial ischemia revisited: basic biological concepts and focus on latest clinical trials," Angiogenesis, vol. 15, no. 1, pp. 1-22, 2012.

[4] H.-J. Kang, H.-S. Kim, S.-Y. Zhang et al., "Effects of intracoronary infusion of peripheral blood stem-cells mobilised with granulocyte-colony stimulating factor on left ventricular systolic function and restenosis after coronary stenting in myocardial infarction: the MAGIC cell randomised clinical trial," The Lancet, vol. 363, no. 9411, pp. 751-756, 2004.

[5] A. Yanagisawa-Miwa, Y. Uchida, F. Nakamura et al., "Salvage of infarcted myocardium by angiogenic action of basic fibroblast growth factor," Science, vol. 257, no. 5075, pp. 1401-1403, 1992.

[6] F. R. Formiga, E. Tamayo, T. Simón-Yarza, B. Pelacho, F. Prósper, and M. J. Blanco-Prieto, "Angiogenic therapy for cardiac repair based on protein delivery systems," Heart Failure Reviews, vol. 17, no. 3, pp. 449-473, 2012.

[7] B. Wang, J. Rosano, D. L. Crabbe, and M. F. Kiani, “Targeted delivery of VEGF to treat myocardial infarction," in Oxygen Transport to Tissue XXXIV, vol. 765 of Advances in Experimental Medicine and Biology, pp. 307-314, Springer, New York, NY, USA, 2013.

[8] J. Kastrup, E. Jørgensen, A. Rück et al., “Direct intramyocardial plasmid vascular endothelial growth factor- $\mathrm{A}_{165}$ gene therapy in patients with stable severe angina pectoris: a randomized double-blind placebo-controlled study: the Euroinject One trial," Journal of the American College of Cardiology, vol. 45, no. 7, pp. 982-988, 2005.

[9] S. Rajagopalan, E. R. Mohler III, R. J. Lederman et al., "Regional angiogenesis with vascular endothelial growth factor in peripheral arterial disease: a phase II randomized, double-blind, controlled study of adenoviral delivery of vascular endothelial growth factor 121 in patients with disabling intermittent claudication," Circulation, vol. 108, no. 16, pp. 1933-1938, 2003.

[10] F. R. Formiga, B. Pelacho, E. Garbayo et al., "Sustained release of VEGF through PLGA microparticles improves vasculogenesis and tissue remodeling in an acute myocardial ischemiareperfusion model," Journal of Controlled Release, vol. 147, no. 1, pp. 30-37, 2010.

[11] T. K. Rosengart, L. Y. Lee, S. R. Patel et al., "Angiogenesis gene therapy: phase I assessment of direct intramyocardial administration of an adenovirus vector expressing VEGF121 cDNA to individuals with clinically significant severe coronary artery disease," Circulation, vol. 100, no. 5, pp. 468-474, 1999.

[12] A. N. McGinn, H. Y. Nam, M. Ou et al., "Bioreducible polymertransfected skeletal myoblasts for VEGF delivery to acutely ischemic myocardium," Biomaterials, vol. 32, no. 3, pp. 942-949, 2011.

[13] C. Wang, X. Bao, X. Ding et al., "A multifunctional selfdissociative polyethyleneimine derivative coating polymer for enhancing the gene transfection efficiency of DNA/polyethyleneimine polyplexes in vitro and in vivo," Polymer Chemistry, vol. 6, no. 5, pp. 780-796, 2015.

[14] P. Linsel-Nitschke and A. R. Tall, "HDL as a target in the treatment of atherosclerotic cardiovascular disease," Nature Reviews Drug Discovery, vol. 4, no. 3, pp. 193-205, 2005.

[15] X. Collet, A. R. Tall, H. Serajuddin et al., "Remodeling of HDL by CETP in vivo and by CETP and hepatic lipase in vitro results in enhanced uptake of HDL CE by cells expressing scavenger receptor BI," Journal of Lipid Research, vol. 40, no. 7, pp. 11851193, 1999.

[16] M. L. Fitzgerald, Z. Mujawar, and N. Tamehiro, "ABC transporters, atherosclerosis and inflammation," Atherosclerosis, vol. 211, no. 2, pp. 361-370, 2010.

[17] M. M. K. Shahzad, L. S. Mangala, H. D. Han et al., "Targeted delivery of small interfering RNA using reconstituted highdensity lipoprotein nanoparticles," Neoplasia, vol. 13, no. 4, pp. 309-319, 2011.

[18] K. C. Vickers, B. T. Palmisano, B. M. Shoucri, R. D. Shamburek, and A. T. Remaley, "MicroRNAs are transported in plasma and 
delivered to recipient cells by high-density lipoproteins," Nature Cell Biology, vol. 13, no. 4, pp. 423-435, 2011.

[19] D. A. Bull, S. H. Bailey, J. J. Rentz et al., "Effect of Terplex/VEGF165 gene therapy on left ventricular function and structure following myocardial infarction: VEGF gene therapy for myocardial infarction," Journal of Controlled Release, vol. 93, no. 2, pp. 175-181, 2003.

[20] Y.-W. Won, A. N. McGinn, M. Lee, K. Nam, D. A. Bull, and S. W. Kim, "Post-translational regulation of a hypoxia-responsive VEGF plasmid for the treatment of myocardial ischemia," Biomaterials, vol. 34, no. 26, pp. 6229-6238, 2013.

[21] C. Benavides-Vallve, D. Corbacho, O. Iglesias-Garcia et al., "New strategies for echocardiographic evaluation of left ventricular function in a mouse model of long-term myocardial infarction," PLoS ONE, vol. 7, no. 7, Article ID e41691, 2012.

[22] T. Simoń-Yarza, E. Tamayo, C. Benavides et al., "Functional benefits of PLGA particulates carrying VEGF and $\mathrm{CoQ}_{10}$ in an animal of myocardial ischemia," International Journal of Pharmaceutics, vol. 454, no. 2, pp. 784-790, 2013.

[23] C. He, Y. Hu, L. Yin, C. Tang, and C. Yin, "Effects of particle size and surface charge on cellular uptake and biodistribution of polymeric nanoparticles," Biomaterials, vol. 31, no. 13, pp. 36573666, 2010.

[24] L. K. Limbach, Y. Li, R. N. Grass et al., "Oxide nanoparticle uptake in human lung fibroblasts: effects of particle size, agglomeration, and diffusion at low concentrations," Environmental Science and Technology, vol. 39, no. 23, pp. 9370-9376, 2005.

[25] X. Bao, W. Wang, C. Wang et al., "A chitosan-graft-PEIcandesartan conjugate for targeted co-delivery of drug and gene in anti-angiogenesis cancer therapy," Biomaterials, vol. 35, no. 29, pp. 8450-8466, 2014.

[26] H. Lv, S. Zhang, B. Wang, S. Cui, and J. Yan, "Toxicity of cationic lipids and cationic polymers in gene delivery," Journal of Controlled Release, vol. 114, no. 1, pp. 100-109, 2006.

[27] Y. Ding, Y. Wang, J. Zhou et al., "Direct cytosolic siRNA delivery by reconstituted high density lipoprotein for target-specific therapy of tumor angiogenesis," Biomaterials, vol. 35, no. 25, pp. 7214-7227, 2014. 

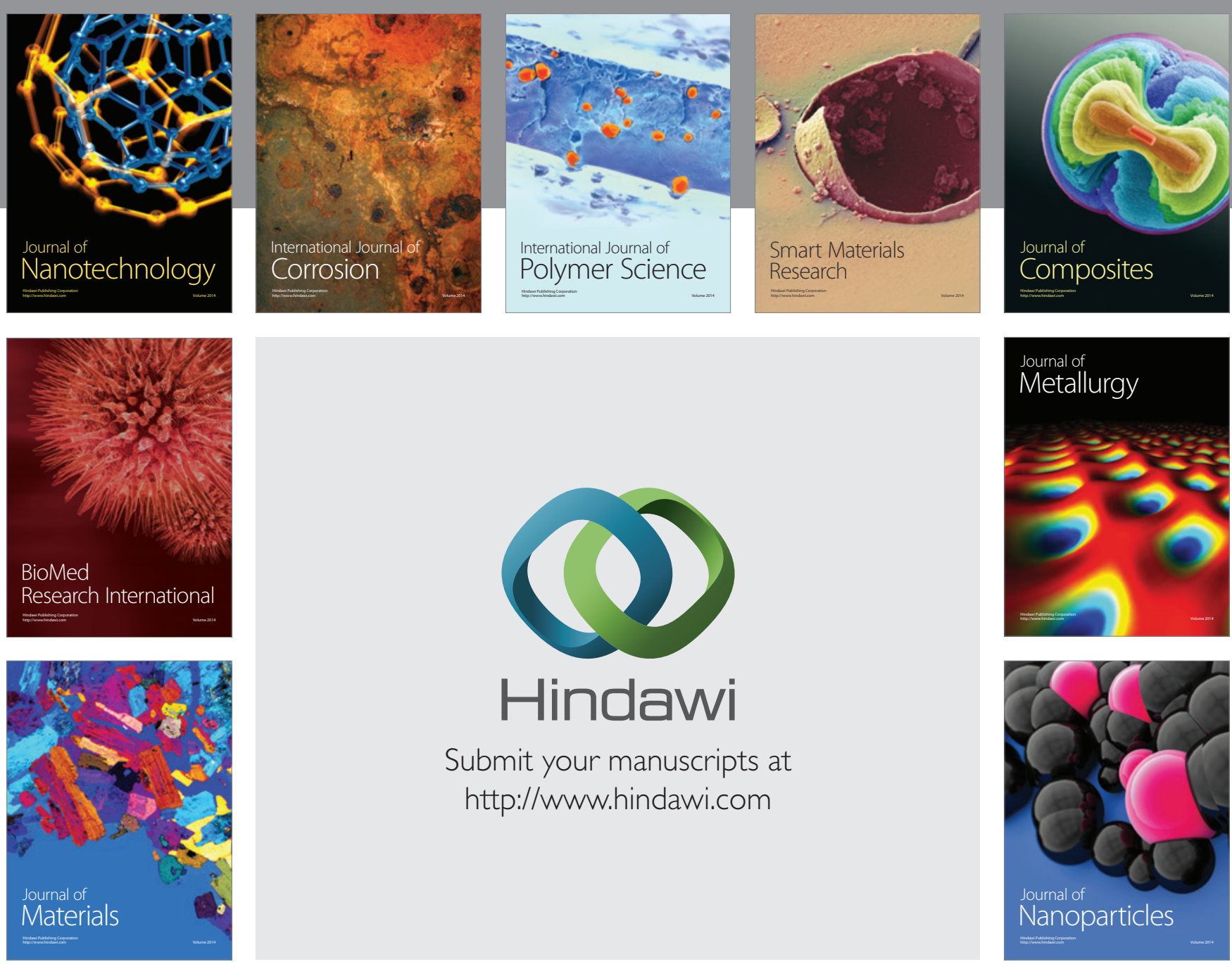

Submit your manuscripts at http://www.hindawi.com
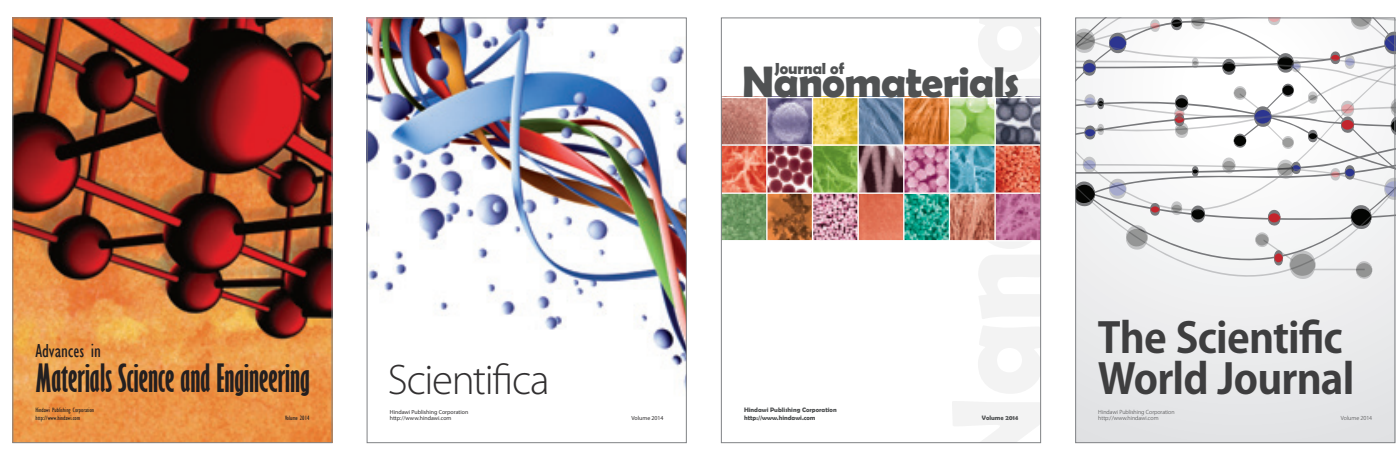

\section{The Scientific World Journal}
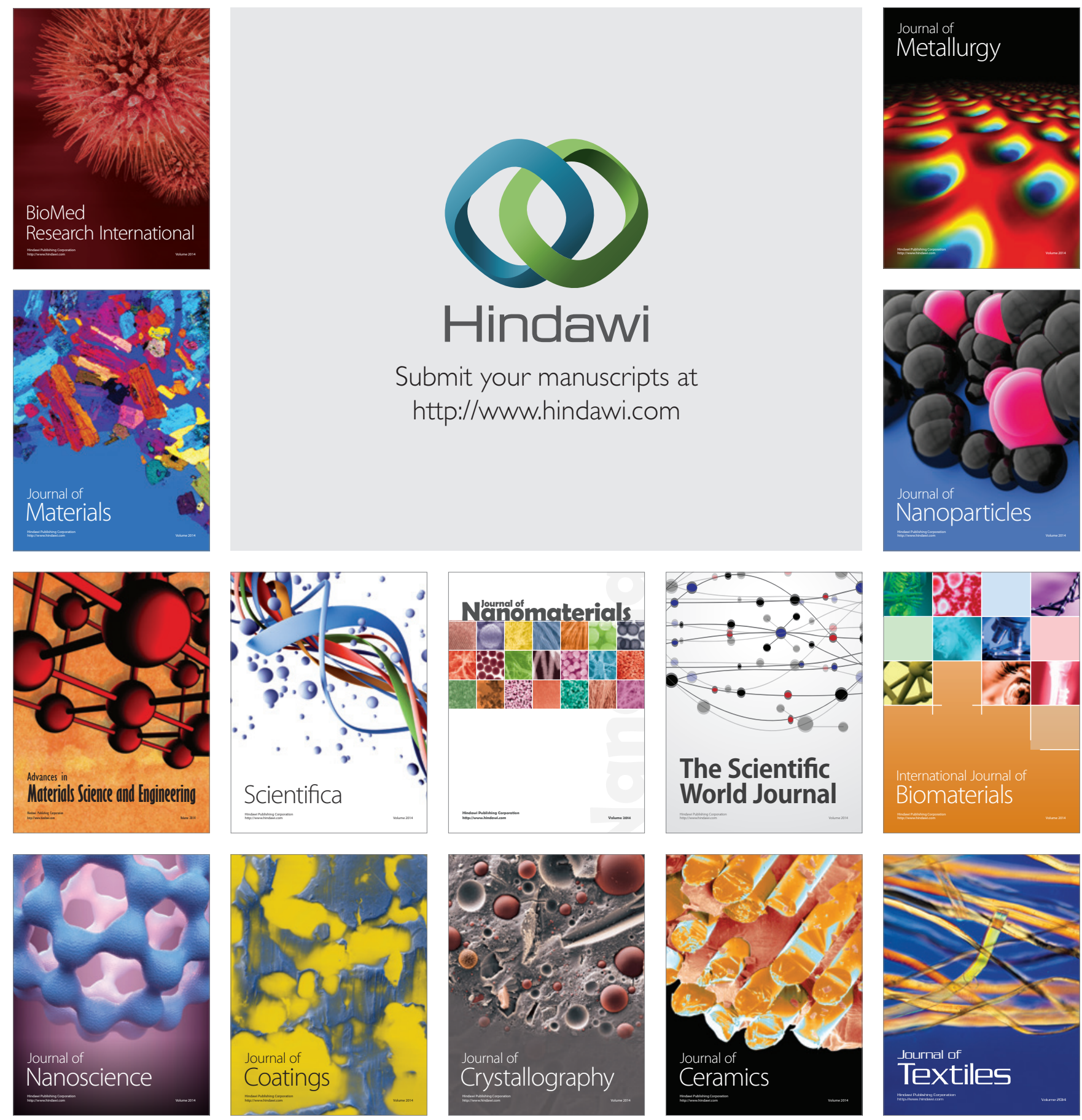